\title{
Design of Vehicle Automation Using Brain Wave
}

\author{
Rakesh T R $\mathbf{R}^{1}$, Sridhar. $\mathbf{N}^{2}$ \\ ${ }^{1}$ M.Tech (VLSI), The Oxford College of Engineering, Bangalore, India \\ ${ }^{2}$ Asst.Professor, The Oxford College of Engineering, Bangalore, India
}

\begin{abstract}
As rate of populace expanding, correspondingly their necessities additionally expanding quickly. Right now individuals completely relying upon vehicles as a method of transportation. Despite the fact that utilizing of vehicles gives points of interest, because of the absence of consideration of drivers amid driving, results in inadvertent issues. Rate of death is more in mischances when contrast with normal passing. Thus with a specific end goal to lessen the unplanned cases we are proposing this framework. The primary work of this anticipates is perusing the cerebrum signals, examining the mind flags and handling for vehicle control. EEG is the fundamental idea utilized as a part of this framework. Neurosky mind wave sensor is utilized as primitive segment to peruse the cerebrum signals. it inside tweaks the sign level and evacuates the clamor. It give consideration and contemplation level of our human cerebrum. Later flags are ordered to various groups; every band of sign is related to capacities such a path, to the point that taking into account consideration and contemplation level the vehicles are controlled consequently.
\end{abstract}

Keywords: vehicles, Brain signals, Brain signals, EEG, Neurosky mind, wave sensor and meditation level

\section{Introduction}

\subsection{Overview of Neurosky brain sense}

NeuroSky is an organization with the trademark "Bio Sensors for Everyone!", and in late 2009 they discharged an EEG gadget named the NeuroSky mentality requiring little to no effort, went for the customer market (Neurosky, n.d.). Dispatched with programming improvement devices, individuals at home can investigate the innovation, and coordinate it with their own configuration and frameworks. What used to be saved for the individuals who had the huge cash to pay for such gear, are presently particularly accessible for any individual who is intrigued, either as a client, an engineer, or both? This is awesome news for quickening BCI research since more individuals can contribute. The methodology for this anticipates is to use the NeuroSky mentality innovation and do only that. Yet, the outlook just gives the cerebrum wave flag, and to decipher and characterize it, a fake neural system will be utilized.
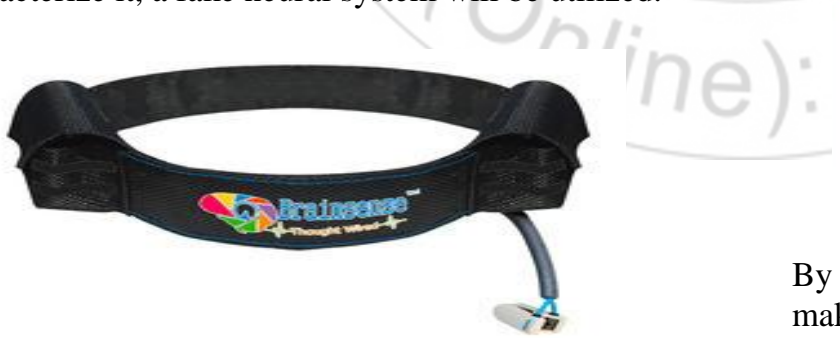

Figure 1.1: Image of Neurosky brainwave sensor

\subsection{Overview of BCI}

The motivation behind this anticipate is to examine and investigate the potential outcomes that exist in the space of Brain-Computer Interfaces, utilizing customer well disposed hardware that have as of late gotten to be accessible on people in general business sector. The field of BrainComputer Interfaces (BCI) is a main impetus for using electroencephalography innovation (EEG), which is the procedure of recording mind movement from the scalp utilizing anodes (See section 3). Before, the fundamental center have been about creating applications in a restorative connection, helping deadened or incapacitated patients to associate with the outer world (Plass-Oude Bos et al., 2010), by mapping mind signs to human intellectual and/or tactile engine capacities.

Further, it is distinguished four different application territories of BCI, some which have been specified in part 3 as of now:

1) Bioengineering applications: Devices with helping purposes for debilitated individuals.

2) Human subject observing: Research and discovery of rest issue, neurological ailments, consideration checking, and/or in general "mental state".

3) Neuroscience examination: ongoing techniques for connecting perceptible conduct with recorded neural signs.

4) Human-Machine Interaction: Interface gadgets between people, PCs or machines.

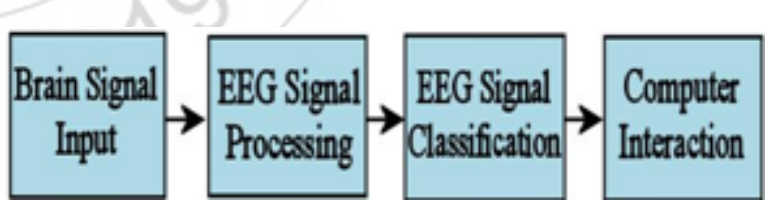

Figure 1.2: Brain-Computer Interface process flow.

By taking BCI to the level of excitement, the inspiration for making more easy to understand, speedier, less expensive and open accessible frameworks will be absolutely different and happen to a much higher need. The focused on gathering of clients is not compelled to use BCI frameworks, and in this way needs better purposes behind needing to, other than it is cool to have the capacity to control your PC with the psyche. Current frameworks don't meet such benchmarks, which is further expounded in the following segment. The propelling believed is that drawing closer this issue from an amusing perspective could cause getting BCIs to such guidelines quicker. 


\subsection{Problem definition and Objectives of Project}

This task ought to incorporate both a hypothetical and a down to earth part. In the hypothetical part, ebb and flow Brain-Computer Interface frameworks and techniques ought to be examined, to make a cutting edge diagram of, and to find out about the area. The emphasis ought to be on the eminent commercialization of EEG items on general society market. In this anticipate, a NeuroSky attitude (EEG gear) is accessible as a test gadget

\section{Objectives of Project}

- Objective 1: to investigate how useful the NeuroSky Mind Wave is in measuring attention levels.

- Objective 2: to investigate how useful the NeuroSky Mind Wave is in measuring relaxation levels.

- Objective 3: to understand the NeuroSkyMindWave's abilities and its limitations for future use.

\section{Literature Survey}

Ye Yuanet.al[1] portrayed a neural-system based computerized epileptic EEG discovery technique which utilizes delay time as the info highlight of a counterfeit neural system.. The outcomes show that the postponement time estimations of EEG signs amid an epileptic seizure get to be bigger than those of typical EEG flags clearly, and after that this wonder is used for computerized epileptic EEG location consolidated with probabilistic neural systems (PNN).

Xiaodong Zhanget.al [2] introduced another expert slave neural system model (just signified as MSNN), whose expert system is two Hopfield systems, and the other slave system is a BP system, separately. After its structure had been creatively planned, the preparation calculation of the MSNN was just talked about. And afterward, a two-channel EEG estimation framework was set up, and the element of the related EEG signals extricated

Karayianniset.al [3] built up a way to deal with distinguish epileptic seizure portions in the neonatal electroencephalogram (EEG) by describing the ghastly components of the EEG waveform utilizing a standard based calculation fell with a neural system. A guideline based calculation screens out short sections of pseudo sinusoidal EEG designs as epileptic in view of components in the force range.

\section{Brief History of Project}

The presence of electrical streams in the mind was initially found in 1875 by an English doctor, Richard Caton. In 1924, Hans Berger (a German researcher) recorded electrical cerebrum movement and utilized the word electroencephalogram (EEG) to portray mind electric possibilities in people. He perceived that "...brain movement changes in a steady and conspicuous way when the general status of the subject changes from unwinding to readiness." (Teplan, 2002). In 1934, Adrian and Matthews distributed a paper which checked the idea of 'human cerebrum waves'.

\subsection{EEG}

To quantify cerebrum action, the electrooculogram(EOG) or the electroencephalograph (EEG) is generally utilized. EOG gives a measure of the distinction in electrical action between the cornea and the retina, and is essentially utilized when measuring eye developments, for example, eye squint rate and eye conclusion. In a paper by Miller in 2001, it was accounted for that constrained exploration had been led into the advantages of utilizing EOG for workload estimation.

- Gamma Waves (>30 Hz): Relate to some senses and memory.

- Beta Waves (13-30 Hz): Focus, active attention, thinking, problem-solving.Beta Waves dominate when the brain is aroused and mentally engaged in activities.

- Alpha Waves (8-13 Hz): Relaxation, meditation, nonarousal, relaxedawareness without any concentration. Alpha Waves can be induced by closing the eyes and relaxing, and abolished by opening the eyes or engaging the brain in activities such as thinking or calculating. In a study by Klimesch et al., it was found that low alpha frequencies relate to attention and that high alpha frequencies relate to some cognitive processes such as memory (Guðmundsdóttir, 2011).

- Theta Waves (4-8 Hz): Drowsiness, deep Relaxation, daydreaming

- Delta Waves (0.5-4 Hz): Deep sleep, unconsciousness.

\subsection{BCI}

A brain computer interface (BCI) is a communication pathway which interprets the user's command from their brainwaves to enable simple tasks to be carried out.

\section{Methodology of Project}

\subsection{Experimental design/procedure}

The testing occurred in the Simulator region at TRL and took roughly 45 minutes for each member. The testing strategy included every member undertaking six psychological errands, as sketched out beneath:

- Task 1) Auditory Verbal Learning Test (AVLT): This includes reviewing a listof random words from memory. This test means to gauge the member's Gamma brainwaves $(>30 \mathrm{~Hz})$, which are connected with some faculties and memory.

- Task 2) Digit Span Test: This includes reviewing a progression of digits in a givenorder. This test additionally expects to quantify the member's Gamma brainwaves $(>30$ $\mathrm{Hz}$ ), which are connected with some faculties and memory.

- Task 3) Snowy Pictures Test (SPT): This includes recognizing pictures fromblurred or bended pictures. This test expects to quantify the member's Beta brainwaves (13-30 Hz), which are connected with the cerebrum being stirred, rationally drew in, engaged and mindful.

- Task 4) The Stroop Test: This is a word-induction test which requires thesubject to name the shading that is shown and not the word. This test was utilized as a part of a comparative study by Crowley et al. (2010) which assessed the NeuroSky'sMindSet headset (a prior variant 


\section{International Journal of Science and Research (IJSR) \\ ISSN (Online): 2319-7064 \\ Index Copernicus Value (2013): 6.14 | Impact Factor (2015): 6.391}

of the MindWave) in measuring consideration and reflection levels. This test additionally means to Beta brainwaves.

- Task 5) The Towers of Hanoi: This numerical diversion/riddle comprises ofthree poles and various distinctive measured circles which the subject needs to move whilst obeying essential tenets. This test was additionally utilized as a part of the study by Crowley et al. (2010) which assessed the NeuroSky'sMindSet headset (a prior variant of the MindWave) in measuring consideration and reflection levels. This test likewise intends to Beta brainwaves

- Task 6) Relaxation CD: This includes the subject sitting in a room, shutting theireyes and listening to an unwinding CD. The test will expect to quantify the member's reflection scores.

\subsection{Hypotheses}

\subsubsection{Hypotheses relating to attention and memory tests}

The hypotheses to be tested include:

H01 The AVLT test will have no effect on the participant's Gamma BrainWaves.

H02 The Digit Span test will have no effect on the participant's Gamma Brain Waves.

H03 The Snowy Pictures Test (SPT) will have no effect on the participant's Beta Brain Waves.

H04 TheStroop Test will have no effect on the participant's Beta Brain Waves.

H05 The Towers of Hanoi test will have no effect on the participant's Beta Brain Waves.

\subsubsection{Hypotheses relating to Relaxation CD}

H06 The Relaxation $C D$ will have no effect on the meditation levels of the participant.

\subsubsection{Hypotheses relating to self-completion questionnaire}

H07 There will be no differences between the relative levels of physiologicaland self-report workload scores.

\subsection{BCI Development: EEG Analysis, FFT and Band Powers}

The Fourier Transformation and extraction of band powers is by far the most ap-plied method for signal processing and analysis (Lotte et al., 2007). The algorithm is based on discrete Fourier transform (DFT) equation 4.1, and by applying that to the EEG signal it makes it possible to separate the EEG rhythms (see section 3.2). Definition of the DFT:

$$
X_{k}=X_{X n e^{i 2 k_{N} n}}{ }^{N 1}
$$

The performance of the DTF is $O\left(N^{2}\right)$, but there is a more efficient algorithm called fast Fourier Transform (FFT), that can compute the same result in only $O(N \log N)$. This is a great improvement and one of the reasons why FFT is the favorable method of analyzing EEG signals, and other waves like sound.

\subsection{Different Approaches for Classifying EEG Signals}

There are five categories that covers the most used algorithms in BCI classification systems, and they are: linear classifiers, nonlinear Bayesian classifiers, nearest neighbor classifiers, neural networks, and a combination of classifiers (Lotte et al., 2007). In all categories there have been achieved good BCI results, except for the nearest neighbor classifiers, which seems to not handle dimensionality very well. However, neural networks are the most popular in BCI research, and are the chosen method to classify in this project also.

\subsubsection{Neural Networks: Multilayer Perceptron}

Multilayer perceptron means that the neural network consists of an input layer, possibly and minimum one hidden layer, and one output layer, as shown in fig-ure 4.3. The output of each node in one layer connects to the input of the next layer, but not within the same layer.

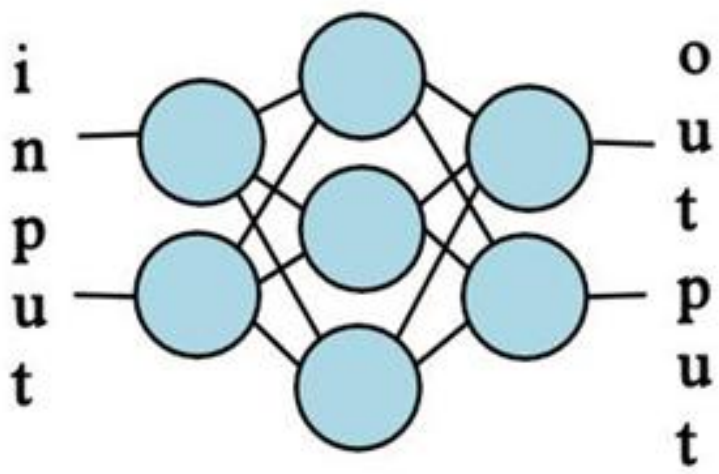

Figure 3.4.1: Neural network

Neurons are also referred to as nodes, and the lines between the nodes are called synapses. The inspiration comes from the network of neurons in the human brain, but very much a simplified version of it. The great strength with neural networks is that they are flexible and almost universal approximates, giving the right setup: Neu-ral networks needs to be trained before it can be used. In case of supervised learn-ing, training sets with sample input and the corresponding output are given to the network, and then an algorithm adjustthe weights of the synapses so that it can map valid input to correct output. A very common algorithm for this use is the backpropagation algorithm. In that case, the neurons look like the one in figure 3.6.1.

Except from the input nodes, all other nodes will have their input determined by the sum of all the incoming synapses. This becomes input to the activation function in the nodes (corresponds to the action potential in biological neurons, see section 3.1), which determine the nodes output: if it "fires" nor not.

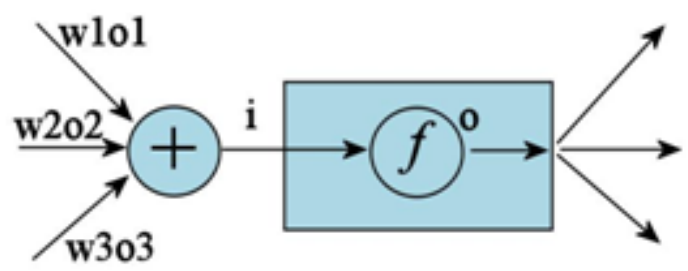

Figure 3.4.2: Neuron in a feed-forward propagation network. $f$ is the activation function 


\section{Back propagation Algorithm}

To understand neural networks better, and how the back propagation works, the algorithm is given in pseudo code below. For more details, please refer to Dario and Mattiussi (2008) and NeuronDotNet (n.d.-a).

Initialize the weights in the network while stopping criterion has reached do for all example e 2 training set do

$\mathrm{O}=$ actual, output(network, e);

propagate forward $\mathrm{T}=$ wanted output for e Calculate error $(\mathrm{T}$

- O) at each neuron in the output layer

Compute Mean Squared Error value; propagate backward

Compute delta weightupdate $_{\text {for all weights }}$

Update all the weights in the network such that the sumsquared value of error is minimized.

end for

end while

The Mean Squared Error (MSE) value is calculated using equation 4.3. This value reflects the effectiveness of the training done so far. The stopping criterion could either be when the MSE has reached an acceptable limit, or when the number of training cycles is attained.
2
$=E[((X))]$
There are several types of activation function commonly used, these are:
- Sigmoid Activation Function: $y=1=(1+\operatorname{Exp}(x))$
- Linear Activation Function: $y=x$
- Logarithmic Activation Function: $y=\log (1+\mathrm{j} x \mathrm{j})$
- Sine Activation Function: $y=\operatorname{Sin}(x)$
- Tanh Activation Function: $y=\operatorname{Tanh}(x)$

\section{Design and Implementation}

At first Neurosky sensor which comprises of dry cathode is set on brow of the driver. Dry terminal peruses the signs from neurons which are situated at the human brow. These signs are then separated to expel the outside clamors and after that tweaked to enhance the sign qualities this is finished by utilizing Thinkgear innovation and eSense meter innovation.

A mind wave sensor comprises of band like thing which comprise of dry anode, thinkgear module, power supply and Bluetooth module. The consideration and contemplation level of the mind signs is recognized by eSense meter and exchanged to the control framework module. In light of consideration level and contemplation level microcontroller controls the vehicle by offering directions to control or to stoop the vehicle.

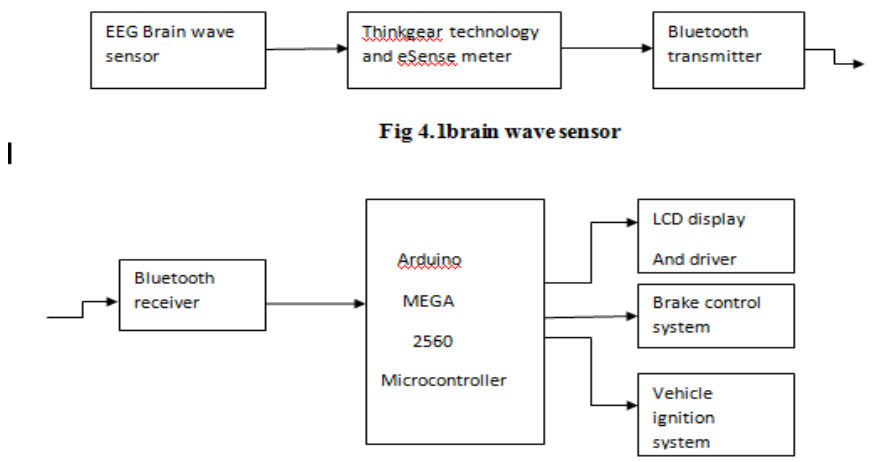

Figure 4.2: Vehicle Control System

Here all the controls are taken by Arduino MEGA microcontroller. Based on instructions which are predefined in a microcontroller necessary actions are taken by microcontroller. This microntroller is an ASIC based thing whiuch is designed for specific applications.

\subsection{EEG Signals}

EEG signals can be collected by many ways. This system using Neurosky sensor to read the data. The brain signals are collected or can be catches by electrode placed in a band like structure named as Neurosky sensor. This is very simple and effective with lower complexity.EEG signals have different amplitudes and different frequency levels.

\subsection{NeuroSky Technology}

\section{i. Brainwaves}

Human brains are made up of large number of neurons. This neurons emits some point of energy during transformation of information, summation of energy released by every neurons results in some signals. These signals are identified by electrode present in Neurosky sensor. Based on this energy signals are classified into different frequency levels as shown in table. 1

\section{ii. ThinkGear}

ThinkGear is the technology used in NeuroSky product that empowers the reading of brainwaves. It includes the sensor that indicates contact and reference points located in the ear clip and sensor that touches the forehead and the on-board chip that processes all of the data named as TGAM. Both the raw brainwaves and the eSense Meters are computed on the ThinkGear chip.

\begin{tabular}{|l|l|l|}
\hline $\begin{array}{l}\text { Brainwave } \\
\text { Type }\end{array}$ & Frequency range & Mental states and conditions \\
\hline Delta & $0.1 \mathrm{~Hz}$ to $3 \mathrm{~Hz}$ & $\begin{array}{l}\text { Deep, dreamless, non-REM sleep, } \\
\text { unconscious }\end{array}$ \\
\hline Theta & $4 \mathrm{~Hz}$ to $7 \mathrm{~Hz}$ & Intuitive, recall, fantasy, imaginary, dream \\
\hline Alpha & $8 \mathrm{~Hz}$ to $12 \mathrm{~Hz}$ & Relaxed, but not drowsy, tranquil, conscious \\
\hline Low Beta & $13 \mathrm{~Hz}$ to $15 \mathrm{~Hz}$ & $\begin{array}{l}\text { Formerly SMR, relaxed yet focused, } \\
\text { integrated }\end{array}$ \\
\hline Midrange Beta & $16 \mathrm{~Hz}$ to $20 \mathrm{~Hz}$ & aware of self \& surroundings, thinking \\
\hline High Beta & $21 \mathrm{~Hz}$ to $30 \mathrm{~Hz}$ & Alertness, agitation \\
\hline \multicolumn{2}{|c|}{ Table4.3. Frequencies Generated By Different Types of Activities in the Brain } \\
\hline
\end{tabular}




\section{International Journal of Science and Research (IJSR) \\ ISSN (Online): 2319-7064}

Index Copernicus Value (2013): 6.14 | Impact Factor (2015): 6.391

\subsubsection{Circuit Diagram of Power Supply \\ +5V POWER SUPPLY}
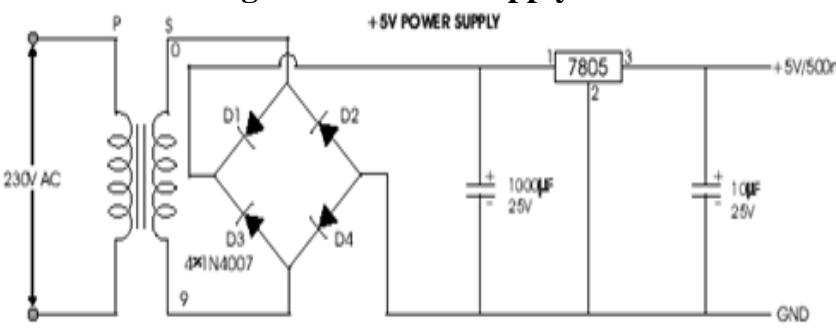

+12 AND - 12V POWER SUPAY

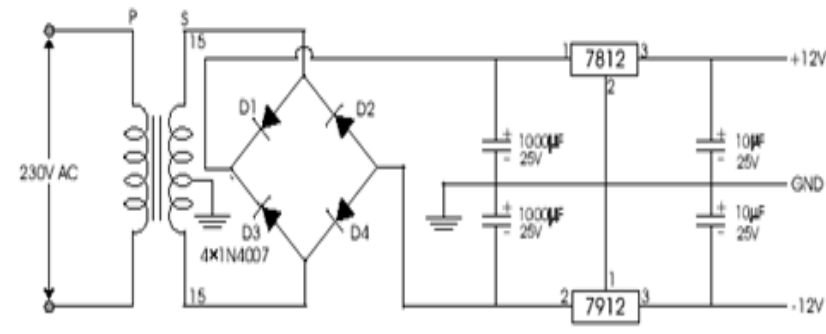

Fig 5.6.1; bleck diagram of power supply

\subsubsection{Working principle}

The AC voltage, typically $220 \mathrm{~V} \mathrm{rms}$, is connected to a transformer, which steps that ac voltage down to the level of the desired DC output. A diode rectifier then provides a fullwave rectified voltage that is initially filtered by a simple capacitor filter to produce a dc voltage. This resulting dc voltage usually has some ripple or ac voltage variation. A regulator circuit removes the ripples and also remains the same dc value even if the input dc voltage varies, or the load connected to the output dc voltage changes.

\section{Results Analysis and Discussion}

\subsection{Experiments and Results}

The data given by brain wave sensor are extracted and compared with the above frequency bands, based on the frequency bands the attention level and meditation level of a person (driver) can be determined. Based on attention value certain functions are allotted. When the person attention level is decreases from higher attention then the driver get alertness about lack of attention and also message will be displayed in vehicle so that surrounding vehicles can be alerted about the drivers lag in attention. If the drivers attention level comes to reach threshold i.e., even after alerting if driver not comes to attention then the system automatically turns off the ignition of the vehicle to avoid accidental cases

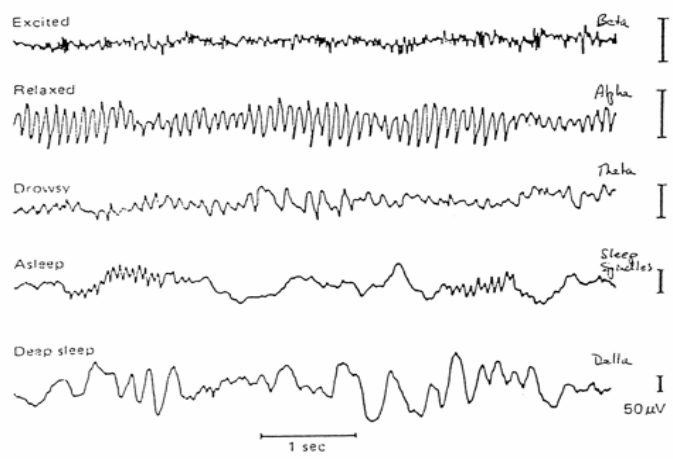

Step1: Extracting the signal using the brainwave sensor.

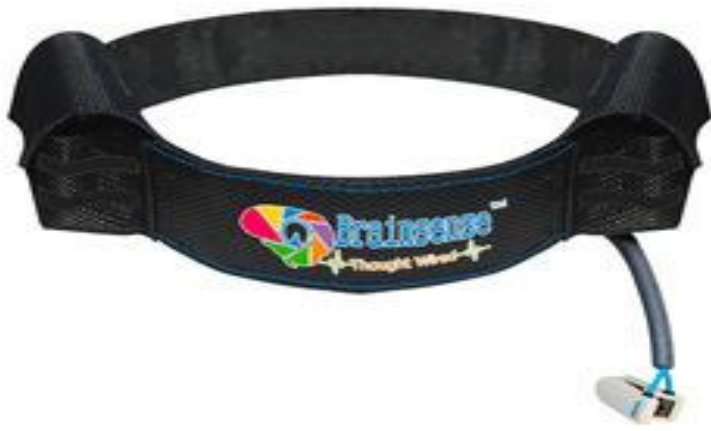

Figure 6.1: Image of brainwave sensor

Step 2: reciving data from the brainwave sensor to vehicle system.

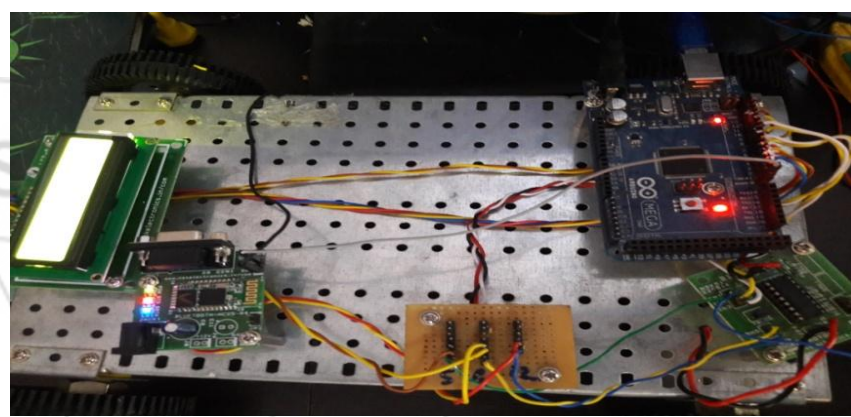

Figure 6.2: Vehicle system

Step 3: If attention level is below 70 it gives alartness

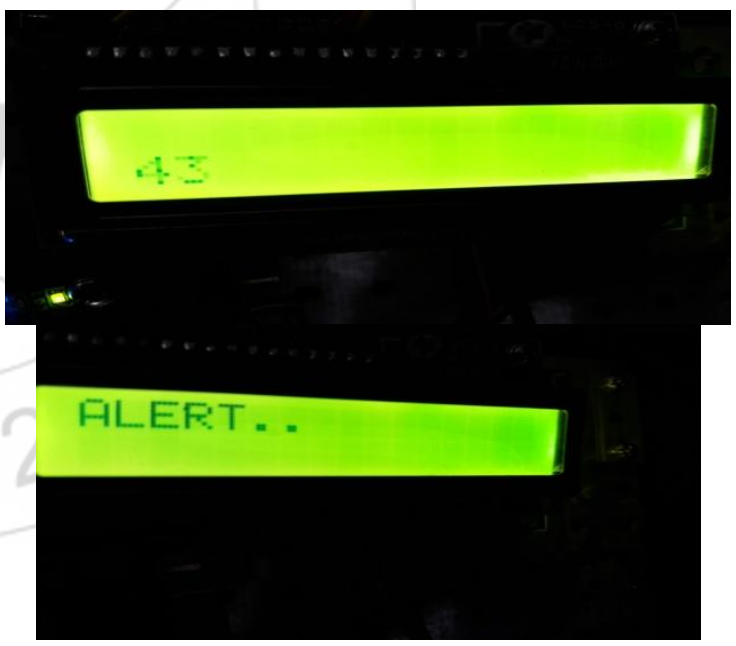

Figure 6.3: attention level

Step 4: when attention level is too poor.



Figure 6.4: Danger level 


\section{Conclusion and Future Work}

In this way this proposed work investigations the condition of driver amid driving by perusing the cerebrum signals. The signs gathered by Neurosky have distinctive abundance and recurrence level. This sign are changed over into parcels and sent to the .Microcontroller investigations the consideration and reflection level and controls the vehicle under poor consideration levels. In this way by controlling vehicles it can spare numerous mishaps and can spare numerous lives.

\section{Future Work}

Going further, implementing this type of devices can reduce the accident, traffic problems. In future these devices can implement in helmet for avoiding the two wheeler accident.
Human Performance in a Real Operating Environment through Real-Time Mental Workload Detection, MIT Press

[12]Lei, S., Welke, S. and Roetting, M. (2009) Driver's Mental Workload Assessment Using EEG Data in a Dual Task Paradigm, National Highway Traffic Safety Administration

[13] Miller, S., 2001 Literature Review - Workload Measures, The University of Iowa

[14] Min, W. and Luo, G. (2009) Medical Applications of EEG Wave Classification, CHANCE, Vol.22, Issue 4, pp.14-20

\section{References}

[1] Ali, Md Z. (2012) EEG-Based Assessment of Driver's Cognitive Response In Virtual Traffic Light Environment, The Faculty of the College of Graduate Studies, Lamar University

[2] American Electroencephalographic Society. (1994). Guidelines for standard electrode position nomenclature.Journal of clinical neurophysiology, 11 , pp.111-113.

[3] Cester, I., Riera, A., Whitmer, D., Dunne, S., SoriaFrisch, A. and Allison, B. (2011) Report on Sensors, Signals and Signal Processing, Future Directions in Brain/Neuronal Computer Interaction (Future BNCI)

[4] Crowley, K., Sliney, A., Pitt, I. and Murphy, D. (2010) Evaluating a Brain-Computer Interface to Categorise Human Emotional Response, 2010 10th IEEE International Conference on Advanced Learning Technologies

[5] Grierson, M. and Kiefer, C. (2011) Better Brain Interfacing for the Masses: Progress in Event-Related Potential Detection using Commercial Brain Computer Interfaces, CHI 2011, May 7-12, 2011, Vancouver, BC, Canada

[6] Guðmundsdóttir, K. (2011) Improving players' control over the NeuroSky Brain-Computer Interface, School of Computer Science, Reykjavik University

[7] Haufe, S., Treder, M.S., Gugler, M.F., Sagebaum, M., Curio, G. and Blankertz, B. (2011) EEG potentials predict upcoming emergency brakings during simulated driving, Journal of Neural Engineering, 8, 056001

[8] Hu, S., Zheng, G. and Peters, B. (2012) Driver fatigue detection from electroencephalogram spectrum after electrooculography artefact removal, IET Intelligent Transport Systems, Vol.7, Issue 1, pp.105-113

[9] Khalilardali, Z., Chavarriaga, R., Gheorghe, L.A. and Millan, J.d.R. (2012) Detection of Anticipatory Brain Potentials during Car Driving

[10] Kim, J.Y., Park, J.B. and Chung, B.J. (2001) Evaluation of Driver's psychophysiological response in general driving using probability density function, Proceedings World Conference on Transport Research

[11] Kohlmorgen, J., Dornhege, G., Braun, M.L., Blankertz, B., Muller, K-R., Curio, G., Hagemann, K., Bruns, A., Schrauf, M. and Kincses, W.E. (2007) Improving 